\title{
Condensation-based routing in mobile ad-hoc networks
}

\author{
Francesco Palmieri $^{\mathrm{a}, *}$ and Aniello Castiglione ${ }^{\mathrm{b}}$ \\ ${ }^{a}$ Dipartimento di Ingegneria dell'Informazione, Seconda Università degli Studi di Napoli, Aversa (CE), \\ Italy \\ ${ }^{\mathrm{b}}$ Dipartimento di Informatica, Università degli Studi di Salerno, Fisciano (SA), Italy
}

\begin{abstract}
The provision of efficient broadcast containment schemes that can dynamically cope with frequent topology changes and limited shared channel bandwidth, is one of the most challenging research topics in MANETs, and is crucial to the basic operations of networks serving fully mobile devices within areas having no fixed communication infrastructure. This problem particularly impacts the design of dynamic routing protocol that can efficiently establish routes to deliver data packets among mobile nodes with minimum communication overhead, and at the same time, ensure high throughput and low end-to-end delay. Accordingly, this work exploits and analyzes an adaptive probabilistic broadcast containment technique based on a particular condensation phenomenon borrowed from Quantum Mechanics and transposed in self-organizing random networks, that has the potential to effectively drive the on-demand route discovery process. Simulation-based performance analysis has shown that the proposed technique can introduce significant benefits on the general performance of broadcast-based reactive routing protocols in MANETs.
\end{abstract}

Keywords: Mobile ad-hoc networks, probabilistic broadcast, Bose-Einstein condensation, reactive routing protocols

\section{Introduction}

The widespread deployment of wireless technologies and mobile computing devices has stimulated a considerable interest in mobile ad-hoc networks (MANETs). These are self-organizing and adaptive wireless communication infrastructures consisting of autonomous mobile nodes, operating in a distributed and asynchronous peer-to-peer fashion, that play both the role of connected hosts and routers by simultaneously running user applications and enabling communications between nodes within their wireless coverage areas through the relay of packet traffic on behalf of them. Such nodes can dynamically move around arbitrarily at specific speeds in any direction, so that the resulting network lacks of any stable communication architecture and management structure and is characterized by a randomly evolving topology in both space and time.

Real-time distributed applications, such as "command and control" in mobile sensor environments [24], communications for battlefield operations and life-critical vehicular interactions or road condition related systems [22], multimedia entertainment, group-working and cooperative strategy playing, multiplayer games, etc., are the most attractive candidates to be used over such network infrastructures due to the

\footnotetext{
${ }^{*}$ Corresponding author: Francesco Palmieri, Dipartimento di Ingegneria dell'Informazione - Seconda Università degli Studi di Napoli, Via Roma 29, I-81031 Aversa (CE), Italy. Tel.: +39 0815010510; Fax: +39 0815037042; E-mail: mailto: francesco. palmieri@unina.it.
} 
enormous potentials offered by this technology in enabling ad-hoc opportunistic real-time interactions among mobile networked entities.

Unfortunately, providing effective real-time services in such a complex and unstable environment may be a really challenging task because of the high degree of dynamism characterizing the involved mobile nodes, together with their energy, connection bandwidth, processing power and memory limitations as long as possible impairments and oddities (interference phenomena, etc.) affecting the wireless channels used to ensure communications and the lack of any kind of central control or hierarchical organization.

Consequently, the routing mechanisms and algorithms used to forward packets from their source to the final destination, via multiple continuously changing node paths, must cope with all the above problems and be extremely lightweight in terms of computational and storage resources requirements. In particular, the lack of a fixed topology makes flooding the fundamental dissemination technique for network-wide broadcast propagation, serving as an unavoidable building block for implementing routing protocols in MANETs. Unfortunately, flooding generates a large amount of redundant packets that may rapidly drive to exhaustion some critical resources such as bandwidth and energy as well as cause contention, collisions, and hence, additional packet loss. On the other hand, reducing the number of redundant broadcasts may reduce the degree of reliability. Thus, we have to cope with the challenge of striking a balance between the introduced level of redundancy and reliability. Probabilistic broadcast techniques, especially adaptive ones, may be effective for selective broadcast containment in all the flooding-based protocols.

Accordingly, some fundamental properties of random peer-to-peer organizations, characterized by an almost totally "flat" scheme, Power Laws and large-scale scalability, have been exploited to model a broadcast-storm resistant flooding scheme that revealed to be particularly effective in evolving ad-hoc networks characterized by competition for links. Here, when new nodes are continuously added to the network, a single node (typically the best connected one) or a few nodes (one for each specific area of coverage) acquire a macroscopic fraction of all the links (i.e. they condense on it). In fact, despite the nonequilibrium nature of these ad-hoc self-organizing networks, whose unstructured topology reflects the competition for links, their dynamic evolution follows the Bose statistics [10] and can experience BoseEinstein condensation [4] phenomena. Starting from these considerations, this work investigates the potential performance improvements that can be achieved in the on-demand route discovery context for reactive ad-hoc routing protocols, by applying adaptive probabilistic broadcast containment techniques based on the exploitation of the aforementioned condensation phenomena "transposed" in the field of dynamic random networks.

The Ad-hoc On-Demand Distance Vector (AODV) routing protocol [18] has been selected in order to assess the effectiveness of the proposed technique, because it is one of the most used and studied routing protocols in the mobile ad-hoc environment and its route discovery facility is widely known to lead to significant delays in networks with a large diameter. Performance evaluation results indicate that the proposed technique enables AODV to achieve an higher data packets delivery ratio and a better reachability, while reducing the routing overhead due to the saved re-broadcasts.

\section{Related work}

In the recent mobile networking literature there is a considerable attention to performance issues in MANET routing $[2,12,13,21]$ and in particular to the problem of limiting the communication overhead associated with broadcast propagation in on-demand route discovery and maintenance [3]. A lot of approaches have been proposed to face with this challenge, often based on probabilistic routing 
mechanisms [20] aiming at properly adjusting the forwarding probability according to local topological considerations in such a way to avoid, if possible, the unnecessary and redundant propagation of broadcast messages. Several broadcasting scenarios have been studied in [17] with the sake of mitigating propagation redundancy by using a fixed probabilistic scheme. In addition, a more dynamic probabilistic on-demand route discovery method has been proposed in [23], where the forwarding probability for route request message is estimated by using the number of duplicate requests received on each node.

In [7], another adaptive probabilistic scheme is proposed, where the probability for a node to retransmit a broadcast packet is directly associated to the local node degree combined with a fixed efficiency parameter controlling the reachability of the broadcast. Similarly, an on-demand route discovery scheme combining traditional probabilistic broadcasting with more specific considerations about the coverage area interested in the broadcast propagation, has been presented in [11], whereas an hybrid approach based on both probabilistic and counter-based propagation has been presented in [16]. Different solutions, alternative to probabilistic broadcasting, have also been exploited, such as the one based on Multi-Point Relays (MPR) selection proposed in [14], or the one based on gossiping and presented in [9].

At the best of our knowledge, the adaptive probabilistic scheme reported in this article is the first experience using concepts derived from Quantum Mechanics, such as the Bose-Einstein condensation, to drive and control the propagation of route discovery messages across large scale MANET infrastructures.

\section{Effective routing in MANETs}

In traditional fixed-topology networks, taking routing decisions is a quite straightforward task: packets to be forwarded are usually sent to the neighbor located on the best path, that is the one with the lowest cost to the final destination. Due to the relatively high reliability of paths/routes, no duplication is needed, and hence each packet on every hop is only sent to a single node. On the contrary, in MANETs' things are completely different and the dynamic nature of nodes, that continuously join and leave the network or change their attachment connections by moving between different coverage areas, may introduce frequent path breaks/failures and recalculations. In this environment, a route consists of an ordered set of intermediate nodes, determined on-the-fly and subject to rapid changes, that transport a packet from source to destination by forwarding it from one node to the next one (see Fig. 1).

Each route is a short-lived object resulting from a continuously evolving topology composition process driven by point-to-point or multi-point ad-hoc peer-to-peer associations dynamically established by the moving nodes. That is, when a new node enters into the transmission range of another one, this creates the possibility of having new routes for a destination being available. The detection of a newly moved or powered on node is implemented through a traditional beaconing mechanism. The neighbor discovery single-hop HELLO messages, propagated in broadcast by each node on all its interfaces and retransmitted at regular intervals, give information regarding the availability of a node as a next hop, allowing neighbor adjacency lists to be dynamically built on all the network nodes. Suppose that a node $X$ moves into the neighborhood (wireless coverage range) of a node $Y$. When the nodes start exchanging HELLO packets, the IP address of $X$ is immediately added to the list of neighbors of $Y$ and vice versa (see Fig. 2). Analogously, when $Y$ detects that periodic HELLO packets are no more received from an adjacent node $X$, it is removed from the neighbor list, assuming that it has moved away or has been powered off.

Thus, establishing and maintaining network connectivity will require greater information exchange efforts and some duplication in traffic relay, leading to a noticeable increase in communication overhead. More precisely, when a packet to be forwarded arrives to a node, there might not be an available path to its destination so that the node may have the necessity of temporarily buffering it while searching 


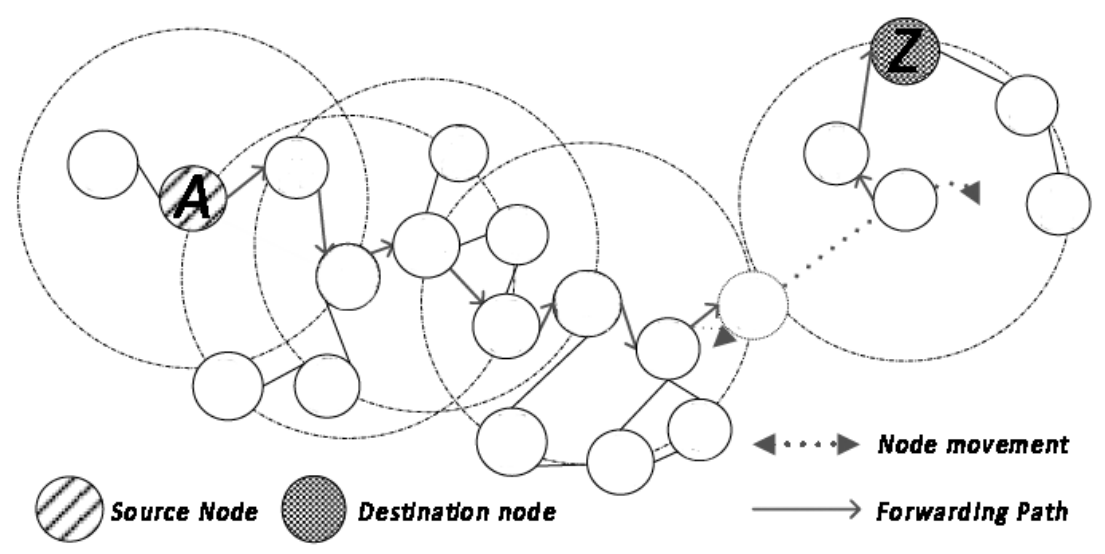

Fig. 1. Hop-by-hop routing in MANETs.
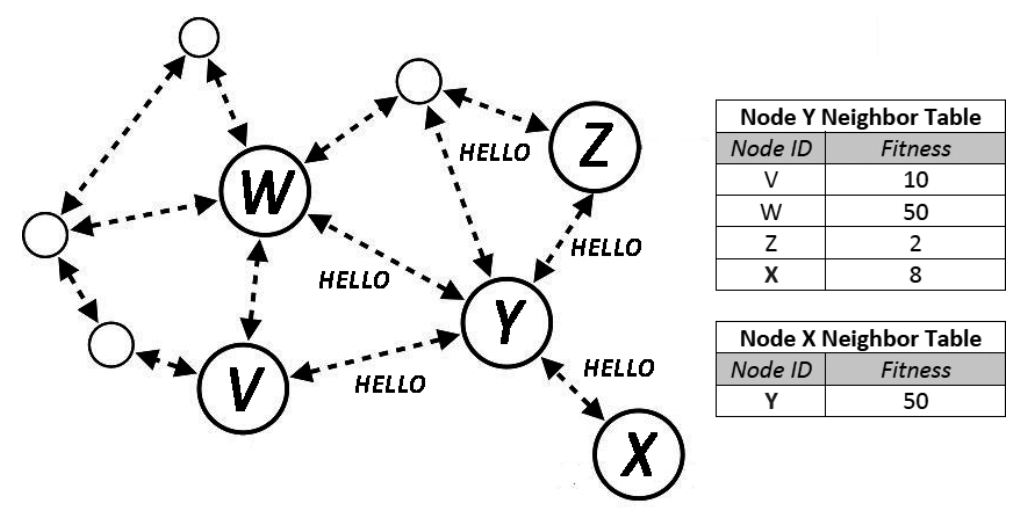

Fig. 2. Neighbor discovery in MANETs.

for a feasible route to the involved destination. The decision on whether forwarding a packet to more than a node is also a concern, since multiple forwarding actions drastically increase the probability of a successful delivery, also in an inherently unreliable environment such as the MANET one.

Such decisions are, unluckily, somewhat difficult to take because only in some situations it could be reasonable to establish an hard threshold and only forward a message to a neighbor when its delivery probability exceeds such threshold. In fact, when excluding a node from the propagation tree, no one can be sure that another neighbor having higher delivery chances will be selected in a realistic time frame. Consequently, in several situations, a greater flexibility is needed during the decision on which neighbor node will be the destination of a broadcast packet. In addition, also the determination of the number of nodes to which a certain packet should be propagated can be an hard choice. In fact, the distribution of packets to the largest possible number of neighbors will extend the probability of a positive delivery, while, simultaneously, it will consume more resources in the entire system. On the contrary, the ability to forward packets only to a limited subset of nodes, and asymptotically to a single one, makes certainly use of few system resources, but decreases the probability of positive delivery and increases the overall end-to-end delay. Furthermore, it should be considered that wireless connections, with respect to their wired counterparts, supply to the involved communicating nodes a lower and less stable bandwidth. 
Consequently, proper extensions are needed at the routing protocols level to support adaptiveness in following the evolving network status as well as to improve the overall efficiency by containing the protocol overhead for maximizing the real bandwidth available for communication.

At the state-of-the-art, MANET routing protocols can be classified into two main classes, depending on their basic operating features:

- Proactive routing protocols strive to keep mobile routes constantly up-to-date as well as to ensure their consistency among the different nodes in a network. In order to have a comprehensive control/view of the entire network, changes in the topology need to be spread over the whole communication infrastructure. Keeping "fresh" routing information for every destination in the network ensures a very limited delay in end-to-end communication between each pair of nodes. Unfortunately, this family of protocols exhibits the drawback of introducing further control traffic to dynamically update route entries that are no more valid (i.e., stale ones). In particular, in scenarios characterized by an high degree of mobility, the implementation of a proactive routing protocol introduces a communication overhead that makes its deployment cost excessively high. For that reason, in presence of ad-hoc communication networks with an high quantity of mobile nodes, reactive routing protocols can be considered a better choice than the proactive one.

- Reactive routing protocols create a route only when it is required, according to an on-demand paradigm. When a new route is needed towards a specific destination, the originating node starts a route discovery process by starting the propagation of a RREQ (Route Request) broadcast packet. The target nodes, or just the intermediate ones (if they own the specific information needed), when replying to the RREQ messages, deliver a backward RREP message (Route Reply) reporting how many intermediate hops are needed to reach the destination. Every node that receives a message of type RREP stores a forward route to the destination node in its routing table. After the complete path has been successfully set up after the source node has received a RREP reply packet, a route management procedure is started to preserve the obtained routing information until the target is no more accessible or the path to it is no longer needed. Reactive protocols require less bandwidth than the proactive ones because they avoid, as possible, the delivery of unneeded recurrent routing information and, at the same time, reduce the network load when just a limited subset of all the available routes is used in a given circumstance. On the other hand, the latency of the on-demand route discovery process can introduce a significant packet delay prior to any communication can take place.

In both cases, broadcasting is used to propagate information that need to be unconditionally disseminated throughout the network (e.g. topology updates or RREQ packets for route discovery). However, RREQ packet does not need to reach all the nodes in the network once a particular path has been discovered that leads to the desired destination. Hence, significant optimization of the routing protocol performance can be achieved by reducing the unnecessary propagation of the above packets with some kind of broadcast control/containment technique. From now on, all the reported considerations will refer to reactive protocols supporting route discovery mechanisms that is the routing technique of choice for this proposal.

Simple flooding, also called pure or blind flooding, is the basic way for implementing the broadcast mechanism, where each node replicates a packet by forwarding it to all its neighboring nodes. Each node receiving a broadcast packet should re-propagate outwards it throughout its neighborhood, eventually stopping the process when every node in the network has already received the broadcast packet. Such 
propagation strategy, despite its inherent simplicity and reliability, presents a significant overhead due to the incontrollable proliferation of delivered messages, with the obvious consequences in terms of redundancy and collisions. Such phenomenon is commonly known as the Broadcast Storm Problem (BSP) [17].

Starting from these considerations, it becomes clear that the propagation mechanisms used in the ondemand route discovery process is fundamental to ensure an acceptable scalability and performance to the involved communication infrastructure. More precisely, the main issue in propagating on-demand routing requests in reactive routing scenarios is directly associated to the minimization of the number of nodes propagating the RREQ broadcast packets while keeping a satisfactory degree of reachability. Clearly, decreasing the number of broadcast retransmissions, immediately saves more bandwidth and reduces the overall node contention and power consumption, with the obvious consequences on the network performance. Thus, a discovery method that provides a good compromise between the minimization of critical resource usage and the maximization of network performance (e.g. throughput and end-to-end delay), is highly desirable.

\subsection{Probabilistic routing}

Several schemes based on probabilistic approaches have been proposed in [7,11,20,23], to overcome the drawbacks associated with simple flooding-based broadcast techniques. Such schemes are really simple to implement, and hence fast and computationally cheap. In fact, they are not adversely affected by node mobility because they require little or no topological information in order to make forwarding/replication decisions and do not need the support of any additional special-purpose hardware.

In traditional approaches based on probabilistic decision techniques, every node involved in broadcast propagation floods a packet throughout its neighborhood, according to a fixed forwarding probability $p$. This can imply that when the value of $p$ is too low, most of the network nodes do not receive the broadcasts or, alternatively, in presence on a excessively high value of $p$, a proliferation of unnecessary redundant transmissions can be experienced. In detail, in presence of $n$ mobile nodes, the maximum (worst case) number of messages needed to ensure the propagation of an RREQ message during a route discovery process will be $p \cdot(n-2)$, when no intermediate node already knows a valid route to the target.

In presence of randomly distributed mobile nodes dynamically establishing ad-hoc connections between each other, several topological regions, characterized by different node density (e.g. sparse and dense regions) and degree of connectivity, can continuously emerge and reorganize themselves within the network. In a highly dense region, multiple nodes may share the same transmission channel coverage being organized in some clustered fashion around some hub/aggregation nodes. In these regions, significant resources can be saved with limited impacts on the broadcast propagation effectiveness, if several nodes do not forward the packet to some randomly chosen neighbors. Conversely, in sparse regions, characterized by a limited degree of coverage, some areas may be completely cut off from broadcast propagation unless the forwarding probability threshold is set high enough. Thus, to cope with these much different situations, such probability need to be adaptively set on all the nodes depending on their local topological and coverage characteristics. Analogously, the distribution of ad-hoc connections within a MANET, and hence the number of connections of each node (its connectivity degree), can significantly influence the broadcast probability, since forwarding the packets towards highest-degree nodes, connected to a large number of peers, greatly enhances the successful delivery chances and reduces the length of the chosen routes/paths throughout the network.

All the approaches based on a static forwarding probability $p$ are adversely affected by unfair broadcast distribution phenomena due to the fixed value of $p$ that is used to take forwarding decisions on each 
node, independently from its specific topological features. Conversely, mobile nodes should be properly categorized according to their communication capabilities (bandwidth, coverage etc.), position and role within the network so that the forwarding probability can be dynamically adjusted to drive the broadcast propagation towards the best connected areas (the denser regions). Furthermore, the limitation of redundant broadcasts should ideally not affect the overall network reachability, and introduce only a minimum dynamic topology management overhead also in presence of a significant node mobility degree, ensuring the needed scalability to the MANET infrastructure.

\subsection{Adaptive probabilistic schemes}

To cope with the above problems, a more adaptive and flexible probabilistic route discovery scheme is needed. It should work by dynamically adjusting the forwarding probability $p$ on each node according to its topological features, mainly characterized by local density considerations. Such density can be easily estimated from the number of adjacent neighbors, that is the node degree.

In typical MANET organizations we can observe the presence of a significant number of nodes connected with a few links (leaf, or lower degree nodes), whereas only a small number of nodes (known as connection hubs) with a great number of links ensure the overall connectivity to all their neighbors. This is due to an implicit preferential attachment mechanism, according to which each node joining the network, in presence of multiple choices and when constrained by the involved transmission technology to a limited number of possible associations, aims at connecting to the neighbors characterized by the best communication capabilities (physical link, aggregated bandwidth, signal health/power, reliability, etc.). Thus, depending on their location, resource availability, link coverage and quality, some nodes tend to physiologically assume the role of hubs or super-nodes within the MANET dynamic peer-to-peer topology, where all the other ones, with lower communication capabilities, will limit their participation to the role of clients or peripheral routers serving a very limited network region. This implies some extent of compliance to the Power Laws, so that the number of hops between nodes is significantly reduced (small-world property) $[1,8]$.

The resulting hierarchy is an extremely important factor to cope with the node heterogeneity, to ensure the scalability of the routing algorithms and to limit the impact of node mobility. In fact, the presence of a few highly connected "focal points" in the network, allows faster propagation of information and hence speeding up both the RREQ and RREP traffic, with significant performance gains for the overall route discovery process. However, due to the former characteristic, if several spoke nodes propagate some information at almost the same time, the involved messages tend to concentrate at the hub node. That is, the nodes with the highest connectivity are subject to most of the search/query traffic. This tendency is likely to increase with the number of connected nodes in the network since also the number of links connected to the hub node increases consequentially.

\subsection{Exploiting condensation phenomena}

When, as the result of moving from a previous location and coverage area to another one, mobile nodes are continuously disconnected and re-added to the network, joining, according to a preferential attachment principle, to $m$ already existing nodes (with $m \geqslant 1$ ) characterized by different connectivity capabilities and hence competing for new communication links/channels, new interesting phenomena can be observed on the evolving network, from which we can take further advantage in the proposed adaptive probabilistic routing scheme. More precisely, we exploit the existence of a close link, presented in [4], between the theory of evolving ad-hoc networks and the equilibrium of a Bose gas (governed 
by the Bose-Einstein statistics [6]) in Quantum Mechanics according to which a dynamically growing network can experience specific clustering/condensation phenomena, due to the competition for links, that resemble the interactions between particles in the above gas. We are interested in modeling the transitions from equilibrium phases, in which all the nodes have the same potential of accepting new connections but some of them (the hubs) gradually tend to form clusters around them by acquiring edges at a higher rate than the others, and a condensation one, in which the best potentially connected nodes condensate most of the new links around them by acquiring a significant portion of all the ad-hoc connections within the network and maintains them over time. This perfectly resembles the analogous phenomenon observable in gases, known as Bose-Einstein condensation [5], in which a large fraction of the bosons occupy the lowest external potential quantum state and the resulting quantum effects become apparent on a macroscopic scale. These considerations and phenomena occurring in complex networks have been formally represented in $[4,19]$ by using a precise model that will constitute the basis for driving the proposed probabilistic routing scheme.

To model the different ability of the network nodes to compete for new ad-hoc connections within their neighborhood, a "fitness" score $\eta_{i}$ (chosen according to a specific distribution $\rho(\eta)$ ) is associated to each node $i \in N$, representing the differences in communication technology, aggregated bandwidth or available channels. In [4], a close mapping from a Bose gas to an evolving network has been defined by assigning an "energy level" $\varepsilon_{i}$ to each node $i$, depending on its fitness $\eta_{i}$ through the relation:

$$
\varepsilon_{i}=-\frac{1}{\beta} \ln \eta_{i}
$$

where $\beta$ is a constant value conditioning its dependency from the fitness $\eta$. In particular, when $\beta$ approaches to zero, all the nodes tend to acquire equal fitness, when instead $\beta \gg 1$ nodes with different "energy" assume very different fitness values. A connection between two nodes $i$ and $j$ characterized by the energy levels $\varepsilon_{i}$ and $\varepsilon_{j}$ and fitness scores $\eta_{i}$ and $\eta_{j}$ corresponds in the mapping to two non-interacting particles placed respectively on their own energy levels. Each new node $i$ joining the network implies the introduction of a new energy level $\varepsilon_{i}$ as long as $2 \cdot m$ new particles in the mapping abstraction. Only $m$ of them remain associated to the energy level $\varepsilon_{i}$, corresponding to the new $m$ outgoing connections established from the node $i$, whereas the other $m$ particles are distributed among the other energy levels (associate the incoming connections to $m$ already existing nodes).

The probability $\Pi_{i}$ that any new node joining the network will connect one of its $m$ links to an already existing node $i$, directly depends on the degree $k_{i}$ and on the "energy" on its level $\varepsilon_{i}$, and is adversely conditioned from the same properties associated to the other nodes $j$ within its neighborhood $N$ :

$$
\Pi_{i}=\frac{e^{-\beta \varepsilon_{i}} k_{i}}{\sum_{j \in N} e^{-\beta \varepsilon_{j}} k_{j}}
$$

In the aforementioned gas/growing network analogy, $\Pi_{i}$ can also be viewed as the probability that a particle lands on level $i$, characterized by an energy $\varepsilon_{i}$ and the number or particles on this level $n\left(\varepsilon_{i}\right)$, closely corresponding to the number of nodes actually connected to the node $i$, is known [4] to follow the Bose statistics:

$$
n\left(\varepsilon_{i}\right)=\frac{1}{e^{\beta\left(\varepsilon_{i}-\mu\right)}-1}
$$

where the aforementioned constant $\beta$ plays, in the Bose gas analogy, the role of an inverse temperature whereas $\mu$ corresponds to the chemical potential. 
Usually, nodes that have been newly created, link to firmly connected nodes acting as "hub". Those nodes are distinguished by the fact of having an high level of fitness. In presence of nodes that are characterized by the same potential in concentrating connections, and hence have the same fitness, the above model coincides with the classic scale free one, described by the Power Laws, leading the network into an almost stable equilibrium.

On the other hand, if the nodes that are already present in the network begin to have a considerable different connection potential, nodes characterized by an higher fitness begin to create links on them in a way which creates clusters and letting to predominate only to those nodes having higher fitness ("fit-get-rich"). In the last phase of the evolution process, it can be observed the condensation of most of the links around the fittest and best connected nodes. In detail, such condensation phenomenon is driven, according to [4], by a fitness distribution $\rho(\eta)$ modeled as:

$$
\rho(\eta)=(1-\eta)^{\lambda}
$$

for each $\lambda>1$.

\subsection{The proposed adaptive algorithm}

We would like to make use of the aforementioned observations and their underlying properties to improve the performance of reactive routing protocols in MANETs by introducing into the AODV protocol a novel highly adaptive probabilistic routing strategy "conditioned" by the condensation phenomenon, assuming that the quantity of "energy" accumulated on a specific node (or its capacity to acquire connections) will indicate how likely this node will be able to deliver a packet to a destination through a reliable and sufficiently short route/path.

To accomplish this, we establish a probabilistic broadcast forwarding strategy based on Eq. (2) simplified as:

$$
p_{i}=\frac{\eta_{i} k_{i}}{\sum_{j \in N} \eta_{j} k_{j}} .
$$

where $p_{i}$ is the probability of forwarding a packet to a specific node $i$, with degree $k_{i}$ and fitness $\eta_{i}$ and $N$ is the complete set of available neighbor nodes with their associated degree $k_{j}$ and fitness $\eta_{j}$ with $j \in N$.

The fitness $\eta_{i}$ assigned to each node can depend on its maximum $b_{i}$ and residual bandwidth $r_{i}$ as long as its signal power $s_{i}$, noise level $n_{i}$ and total/residual number of channels (respectively $c_{i}$ and $a_{i}$ ) as defined in the following equation:

$$
\eta_{i}=\frac{r_{i} \log \left(b_{i}\right) \cdot a_{i} \log \left(c_{i}\right) \cdot s_{i}}{n_{i}}
$$

The above criteria also derive from the consideration that, in the typical preferential attachment model, new nodes strive to establish new connections with nodes with higher degree and fitness, and hence these properties jointly determine the attractiveness of a node. Consequently, as the node fitness changes, so does its role in MANET routing, so that the fittest nodes are those that have greater chances to successfully respond (with RREP messages) to route requests queries (RREQ messages) in the route discovery process. 
The whole probabilistic forwarding strategy, to be adopted on each mobile node for RREQ message delivery in the AODV routing protocol, with the sake of containing the adverse effects of broadcasts during the route discovery process, and driving the RREQ forwarding towards the fittest and best connected nodes, is described in detail by using pseudo-code in Algorithm 1.

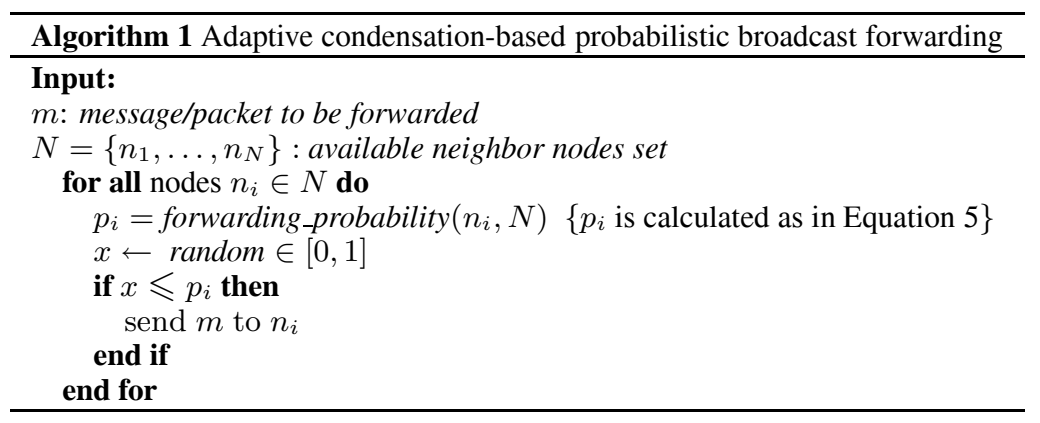

Clearly, as the number of retransmissions required for broadcasting RREQ messages is reduced, more bandwidth is saved and also contention and node power consumption are reduced, by improving overall MANET performance.

\section{Performance evaluation}

The performance and effectiveness of the proposed scheme has been analyzed by using discrete event simulation. The NS-2 simulator [15] has been used in all the evaluation experiments, where the mobile nodes have been modeled as homogeneous (i.e. with the same transmission range and interfaces, simulating the default Lucent's WaveLAN $802.11 \mathrm{~b}$ card with a $2 \mathrm{Mb} / \mathrm{sec}$ bit rate), and the wireless channel is fully shared and can be accessed by any node at random times. Since the $802.11 \mathrm{~b}$ MAC specification is based on CSMA/CA, broadcast collision, control is limited to basic collision avoidance at the carrier sensing level and thus some collisions in broadcast packet transmission may take place.

Several variable CBR traffic loads, relying on UDP transport and with 512 byte packet size, have been used in the analysis, within the context of a random waypoint mobility model in a square field of $1000 \times$ $1000 \mathrm{~m}^{2}$ and a pause time of 0 seconds corresponding to continuous motion of the mobile nodes (with $20 \mathrm{~m} / \mathrm{s}$ maximum nodal speeds). Each simulation was run for 100 seconds. Unicast AODV routing with link layer support and HELLO messages enabled (needed to build the neighbor lists) has been used to discover all the destination nodes solicited by the test traffic. A modified protocol version (referred to as C-AODV in the figures), optimized to reduce the number of retransmission for the route request (RREQ) messages by using the previously presented condensation based broadcast containment techniques, has been compared against the traditional AODV implementation based on blind flooding.

In detail, the above performance comparisons have been performed in two different scenarios, whose goal was to study how the effectiveness of the proposed scheme is affected by the prevailing network conditions such as traffic load and node density:

- In the first one, a single random mobile ad-hoc network consisting of 100 nodes has been solicited with several variable traffic loads ranging from 10 to $10032 \mathrm{Kbps}$ CBR connections.

- In the second one, the network density has been varied from 50 to 150 nodes placed randomly on the aforementioned area, solicited by using a fixed $32 \mathrm{Kbps}$ CBR load of 50 connections. 


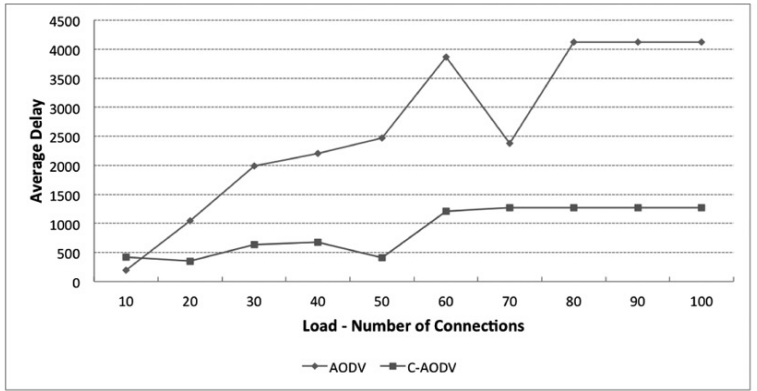

(a) Average Delay

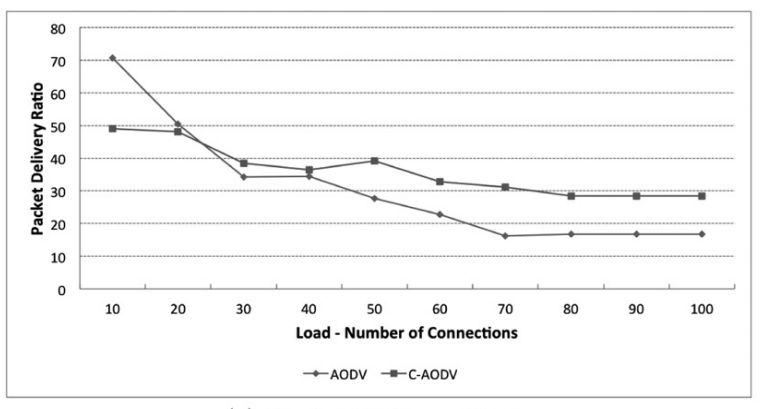

(c) Packet Delivery Ratio

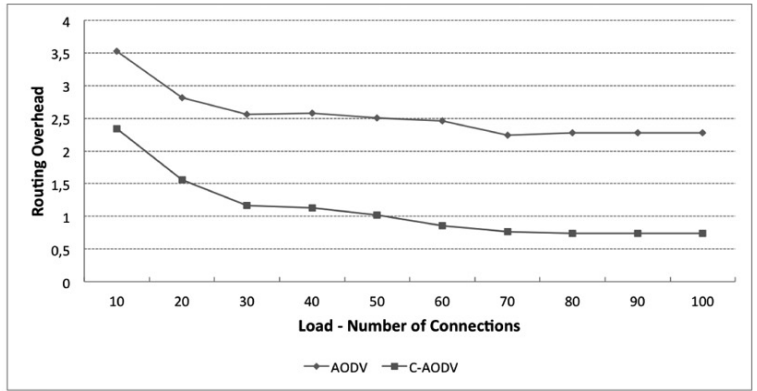

(b) Routing Overhead

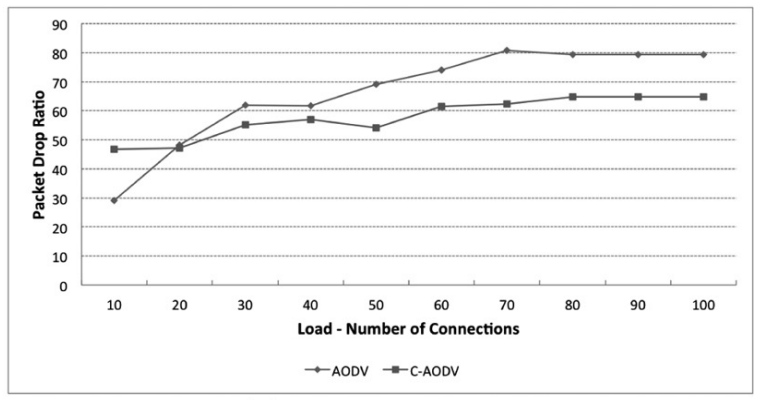

(d) Packet Drop Ratio

Fig. 3. Performance results (a), (b), (c), (d) for a 100-nodes network and load varying from 10 to 100 connections.

In both the scenarios, the metrics used to evaluate the performance of the protocol included:

- The average End-to-end Delay, that is the interval between the instant a source generates a packet and the time at which the destination receives the packet;

- The Routing Overhead calculated as the sum of all routing packets transmitted during the simulation, or normalized routing load. For packets sent over multiple hops, each transmission over one hop is counted as one transmission;

- The Packet Delivery Ratio, representing the percentage of sent packets that have been correctly received;

- The Packet Drop Ratio, representing the percentage of sent packets that have been dropped.

The main result of all the simulation experiments is that the condensation-based protocol version (C-AODV) almost always outperform the conventional one. The improvements in end-to-end delay become progressively more appreciable when the connection load increases (compare Fig. 3(a) with Fig. 4(a)) whereas the gain observable in the reduction of Routing Overhead varies almost linearly with both the load and network density but its growth rate is significantly affected by the network dimensions and adversely conditioned by the increasing load (compare Fig. 3(b) with Fig. 4(b)). The benefits in packet delivery performance and packets dropped become evident only when the network load grows over 20 connections or more (see Figs 3(c) and 3(d)), whereas it generally maintains a slightly better performance for low density as long as high density networks (see Figs 4(c) and 4(d)).

\section{Conclusions}

A new broadcast containment strategy, where the forwarding probability at a node is dynamically computed based on its local density criteria and driven by condensation phenomena similar to those observable 


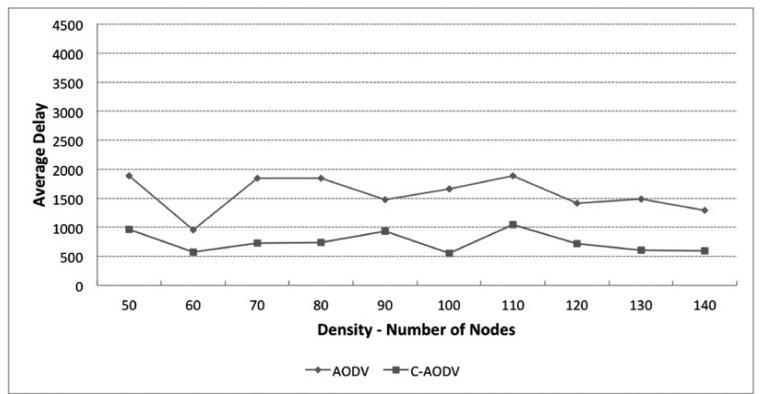

(a) Average Delay

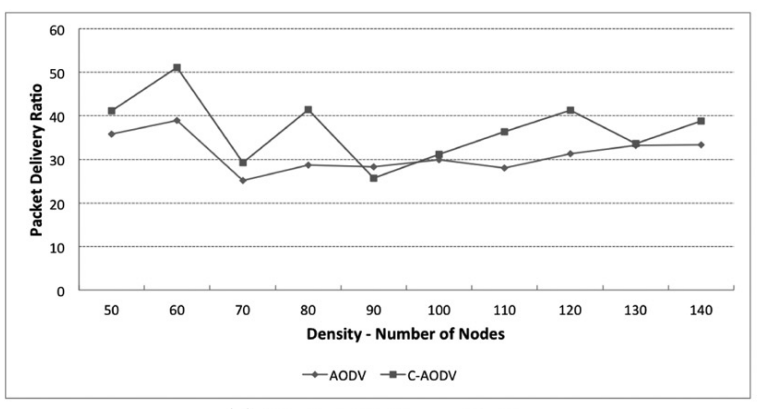

(c) Packet Delivery Ratio

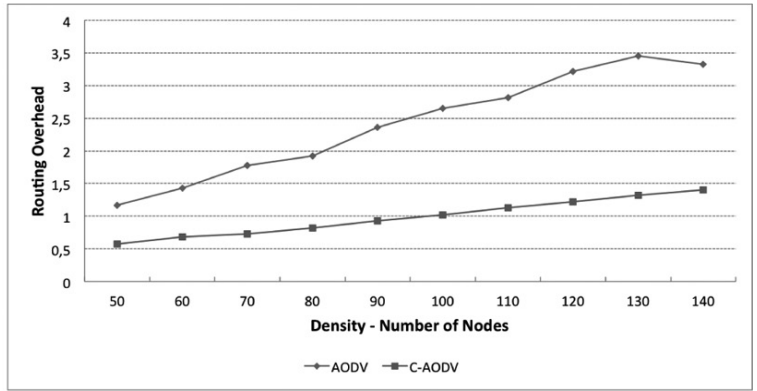

(b) Routing Overhead

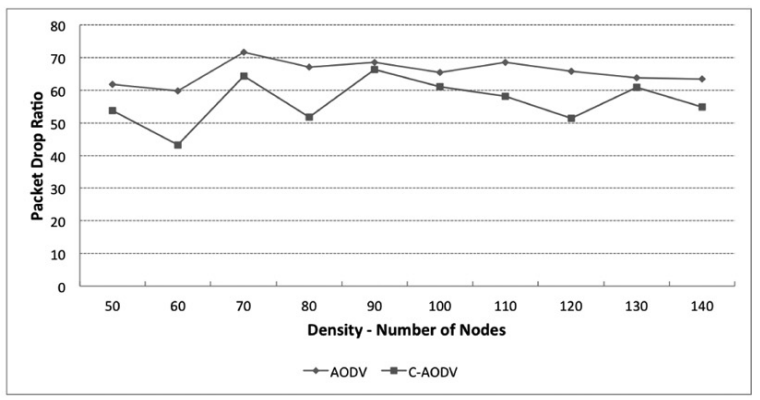

(d) Packet Drop Ratio

Fig. 4. Performance results (a), (b), (c), (d) for a 50-connections load on networks varying from 50 to 140 nodes.

in a Bose gas in quantum mechanics, has been proposed and introduced within the route discovery framework of the AODV MANET routing protocol. The performance of the resulting condensation-based enhanced scheme (C-AODV) has been compared against the traditional one based on blind-flooding. The observed simulation results have shown that, for all the considered network densities and for almost all the offered loads, the proposed scheme outperforms the traditional blind-flooding based AODV routing protocol in terms of reduced routing overhead, packet delivery performance, end-to-end delay, and the achieved benefits become more appreciable when the number of connections or active nodes grows. The success of the presented adaptive probabilistic strategy is due to its ability of striking a good balance between exploration (expanding the RREQ propagation to specific areas of the search space that can bring to promising solutions) and exploitation (considering past experience factors and traffic properties to drive the search) within the network discovery/broadcast propagation process throughout the mobile ad-hoc network.

\section{References}

[1] A.-L. Barabási and R. Albert, Emergence of scaling in random networks, Science 286(5439) (15 October 1999), 509-512.

[2] A. Barolli, E. Spaho, L. Barolli, F. Xhafa and M. Takizawa, QoS routing in ad-hoc networks using GA and multi-objective optimization, Mobile Information Systems 7(3) (2011), 169-188.

[3] S. Basagni, I. Chlamtac, V.R. Syrotiuk and B.A. Woodward, A Distance Routing Effect Algorithm for Mobility (DREAM), In MOBICOM, pages 76-84, 1998.

[4] G. Bianconi and A.-L. Barabási, Bose-Einstein Condensation in Complex Networks, Phys Rev Lett 86 (Jun 2001), 5632-5635.

[5] C.C. Bradley, C.A. Sackett, J.J. Tollett and R.G. Hulet, Evidence of Bose-Einstein Condensation in an Atomic Gas with Attractive Interactions, Phys Rev Lett 75 (Aug 1995), 1687-1690.

[6] A.H. Carter, Classical and Statistical Thermodynamics, Prentice Hall, Upper Saddle River, NJ, 2001. 
[7] J. Cartigny and D. Simplot, Border Node Retransmission Based Probabilistic Broadcast Protocols in Ad-Hoc Networks, In Proceedings of the 36th Annual Hawaii International Conference on System Sciences (HICSS'03) - Track 9 - Volume 9, HICSS '03, Washington, DC, USA, 2003. IEEE Computer Society, pages 303-312.

[8] M. Faloutsos, P. Faloutsos and C. Faloutsos, On power-law relationships of the Internet topology, SIGCOMM Comput Commun Rev 29 (August 1999), 251-262.

[9] Z.J. Haas, J.Y. Halpern and L. Li, Gossip-based ad hoc routing, IEEE/ACM Trans Netw 14(3) (2006), 479-491.

[10] K. Huang, Statistical mechanics, Wiley, New York, NY, USA, 1987.

[11] J.-S. Kim, Q. Zhang and D.P. Agrawal, Probabilistic broadcasting based on coverage area and neighbor confirmation in mobile ad-hoc networks, In Global Telecommunications Conference Workshops, 2004. GlobeCom Workshops 2004. IEEE, Nov. -3 Dec. 2004, pages 96-101.

[12] Y.-S. Kim, Y.-S. Shim and K.-H. Lee, A cluster-based web service discovery in MANET environments, Mobile Information Systems 7(4) (2011), 299-315.

[13] E. Kulla, M. Hiyama, M. Ikeda, L. Barolli, V. Kolici and R. Miho, MANET performance for source and destination moving scenarios considering OLSR and AODV protocols, Mobile Information Systems 6(4) (2010), 325-339.

[14] T.-H. Lin, H.-C. Chao and I. Woungang, An enhanced MPR-based solution for flooding of broadcast messages in OLSR wireless ad hoc networks, Mobile Information Systems 6(3) (2010), 249-257.

[15] S. McCanne and S. Floyd, The network simulator - ns-2. http://www.isi.edu/nsnam/ns/.

[16] A. Mohammed, M. Ould-Khaoua and L.M. Mackenzie, An Efficient Counter-Based Broadcast Scheme for Mobile Ad Hoc Networks, In Katinka Wolter, editor, EPEW, volume 4748 of Lecture Notes in Computer Science, Springer, 2007, pages $275-283$.

[17] S.-Y. Ni, Y.-C. Tseng, Y.-S. Chen and J.-P. Sheu, The Broadcast Storm Problem in a Mobile ad hoc Network, In MOBICOM, 1999, pages 151-162.

[18] C.E. Perkins, E. Belding-Royer and S. Das, Ad-hoc On-Demand Distance Vector (AODV) Routing, IETF RFC 3561, 2003.

[19] A. Réka and A.-L. Barabási, Statistical mechanics of complex networks, Rev Mod Phys 74 (Jan 2002), 47-97.

[20] Y. Sasson, D. Cavin and A. Schiper, Probabilistic broadcast for flooding in wireless mobile ad hoc networks, In Wireless Communications and Networking, 2003. WCNC 2003. 2003 IEEE, volume 2, March 2003, pages 1124-1130.

[21] E. Spaho, L. Barolli, G. Mino, F. Xhafa, V. Kolici and R. Miho, Implementation of CAVENET and its usage for performance evaluation of AODV, OLSR and DYMO protocols in vehicular networks, Mobile Information Systems 6(3) (2010), 213-227.

[22] J. Zhang, C. Chen and R. Cohen, A Scalable and Effective Trust-Based Framework for Vehicular Ad-Hoc Networks, J Wireless Mobile Networks, Ubiquitous Computing, and Dependable Applications 1(4) (2010), 3-15.

[23] Q. Zhang and D.P. Agrawal, Dynamic probabilistic broadcasting in MANETs, J Parallel Distrib Comput 65(2) (2005), 220-233.

[24] T.A. Zia and A.Y. Zomaya, A Lightweight Security Framework for Wireless Sensor Networks, J Wireless Mobile Networks, Ubiquitous Computing, and Dependable Applications 2(3) (2011), 53-73.

\begin{abstract}
Francesco Palmieri is an assistant professor at the Engineering Faculty of the Second University of Napoli, Italy. His major research interests concern high performance and evolutionary networking protocols and architectures, routing algorithms and network security. Since 1989, he has worked for several international companies on nation-wide networking-related projects and, from 1997 to 2010 he has been the Director of the telecommunication and networking division of the Federico II University, in Napoli, Italy. He has been closely involved with the development of the Internet in Italy as a senior member of the Technical-Scientific Advisory Committee and of the CSIRT of the Italian NREN GARR. He has published more that 70 papers in leading technical journals/conferences and currently serves as Editor-in-Chief of an international journal and is part of the editorial board of several other ones.
\end{abstract}

Aniello Castiglione joined the Dipartimento di Informatica ed Applicazioni "R. M. Capocelli" of Università di Salerno in February 2006. He received a degree in Computer Science and his Ph.D. in Computer Science from the same university. He is a reviewer for several international journals (Elsevier, Hindawi, IEEE, Springer) and he has been a member of international conference committees. He is a Member of various associations, including: IEEE (Institute of Electrical and Electronics Engineers), of ACM (Association for Computing Machinery), of IEEE Computer Society, of IEEE Communications Society, of GRIN (Gruppo di Informatica) and of IISFA (International Information System Forensics Association, Italian Chapter). He is a Fellow of FSF (Free Software Foundation) as well as FSFE (Free Software Foundation Europe). For many years, he has been involved in forensic investigations, collaborating with several Law Enforcement agencies as a consultant. His research interests include Data Security, Communication Networks, Digital Forensics, Computer Forensics, Security and Privacy, Security Standards and Cryptography. 

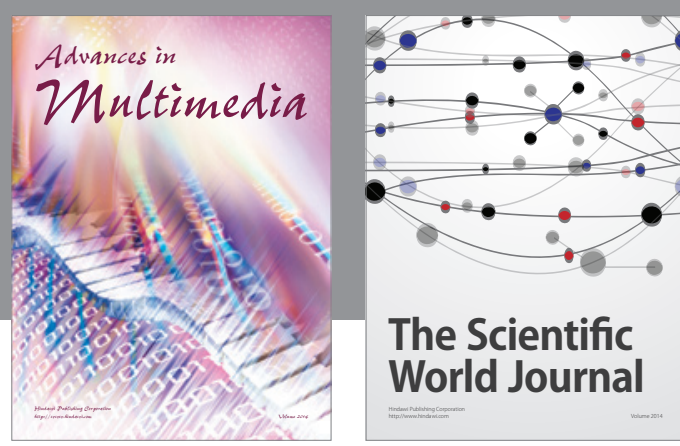

The Scientific World Journal
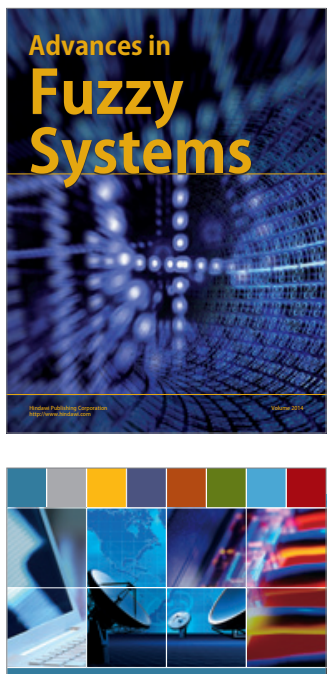

Computer Networks and Communications
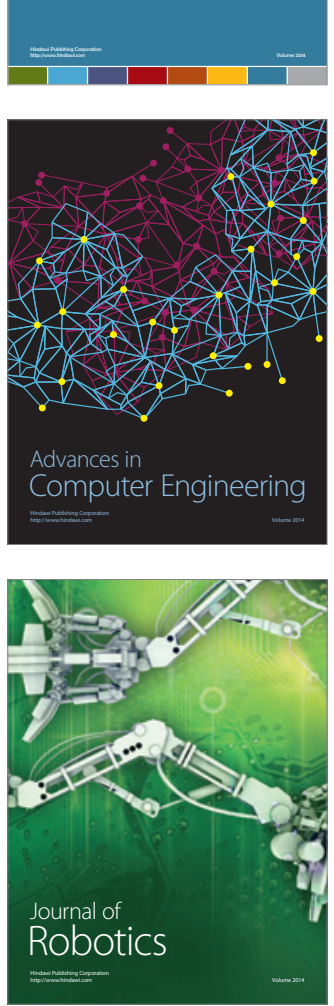
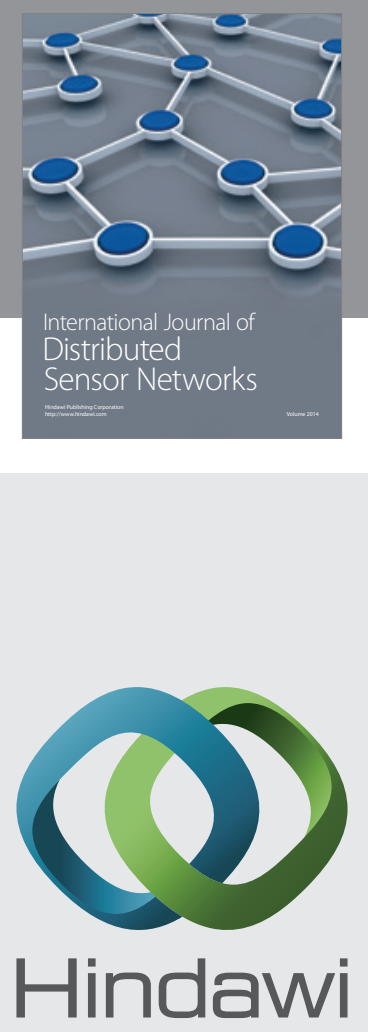

Submit your manuscripts at

http://www.hindawi.com
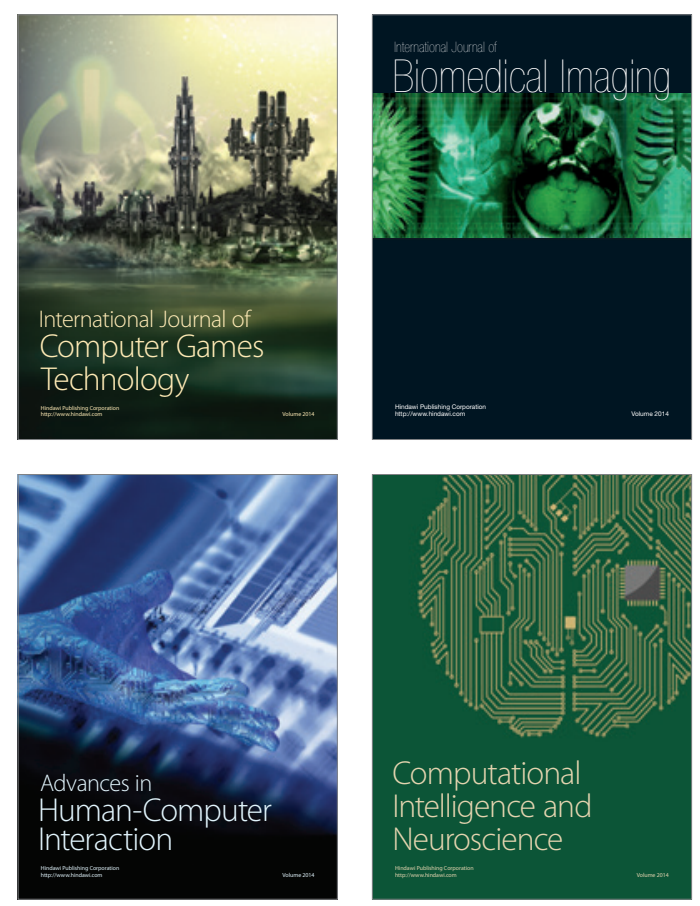
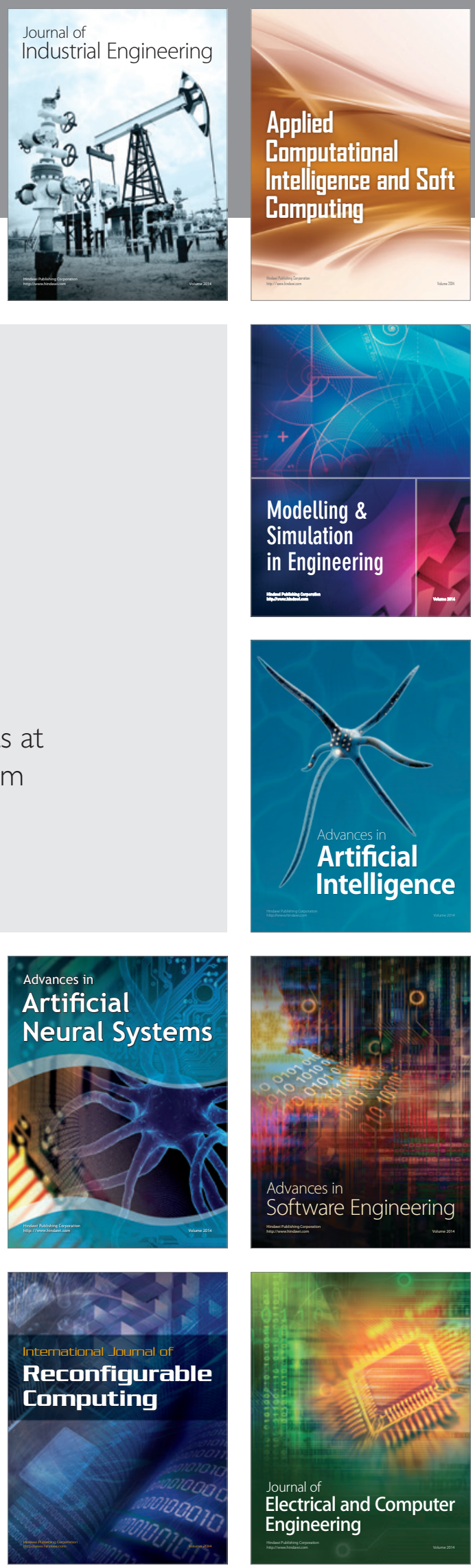